\title{
CT-Urography: Comparison of different methods for increasing the intra-abdominal pressure
}

\author{
Lena Hage*1, Daniel Boll², Philipp Brantner ${ }^{2}$, Georg Bongartz ${ }^{2}$, Silke Potthast ${ }^{3}$ \\ ${ }^{1}$ Department of Diagnostic and Interventional Radiology, Klinikum Darmstadt, Darmstadt, Germany \\ ${ }^{2}$ Department of Radiology and Nuclear Medicine, University Hospital Basel, Basel, Switzerland \\ ${ }^{3}$ Institute of Radiology, Spital Limmattal, Schlieren, Switzerland
}

Received: February 10, 2018

Accepted: March 1, 2018

Online Published: March 5, 2018

DOI: $10.5430 /$ ijdi.v5n $1 \mathrm{p} 25$

URL: https://doi.org/10.5430/ijdi.v5n1p25

\begin{abstract}
Objective: Investigating the influence of increasing the intraabdominal pressure by Valsalva maneuvers and/or abdominal compression to reach best visualization of the entire urinary tract in computed tomography urography (CTU).

Methods: After split-bolus technique, Valsalva maneuvers, compression or a combination of both were applied in 60 patients before late phase images were acquired. The degree of opacification and distension of three segments of the urinary tract were evaluated.

Results: After split-bolus CT no significant difference among the groups regarding distention and opacification. A significant increase in distension and opacification was found during Valsalva and the combination of Valsalva and compression.

Conclusions: A CTU protocol including a late phase scan with prior application of compression, Valsalva maneuvers or a combination of both showed beneficial effects for the distension and opacification, a significant difference was found by taking location as a covariate.
\end{abstract}

Key Words: CT urography, Opacification, Distension, Split-bolus CT

\section{INTRODUCTION}

The ureters are tubes layered by transitional cells, their diameter is ranging from $3 \mathrm{~mm}$ to $7 \mathrm{~mm}$. The ureters have additional smooth muscle fibers in the more distal part to transport the urine from the kidneys to the urinary bladder by peristalsis. These peristaltic contractions take place every 20 to 25 seconds. The contractile waves lead to an almost complete occlusion of the ureter. An additional type of transport is by a hydrostatic pressure gradient in a wide-open ureter occurring during larger urine flow. The small diameter size and the almost complete occlusion during peristaltic waves complicate imaging. To detect pathologies occurring within the lumen of the ureters the aim of imaging is to depict the entire ureter distended with contrast. In former days imaging the urinary collecting system and the ureter was performed by intravenous urography (IVU). ${ }^{[1]}$ This technique is limited by difficulties in presenting the entire ureter in one single image.

Nowadays, computed tomography (CT) is used as first choice imaging for the evaluation of the kidney and urinary tract system, performed as computed tomography urography (CTU). However, currently there are no standard protocols for CTU which ensure optimal visualization of the entire upper urinary tract through contrast opacification and distension of the

\footnotetext{
* Correspondence: Lena Hage; Email: lena.hage@ gmail.com; Address: Department of Diagnostic and Interventional Radiology, Klinikum Darmstadt, Darmstadt, Germany.
} 
urinary tract collecting system. To improve distension and opacification of the upper urinary tract several procedures are established and used during IVU and CTU. Some aim to increase intraabdominal pressure which has been mentioned as early as 1930, ${ }^{[2]}$ for example by performing Valsalva maneuvers or external abdominal compression. ${ }^{[3-5]}$ To our knowledge no previous study compared the effect of Valsalva maneuvers and external abdominal compression or a combination of both on urinary tract opacification and distension, when applied after the nephrographic and excretory phase in late phase CT imaging.

\section{MethodS}

\subsection{Subjects and study design}

Institutional review board approved the study protocol. In our institute, CTU has been the standard type of examination for patients presenting with painless microscopic hematuria to evaluate potential causes of obstruction like stones, neoplasms, blood clots or inflammation in the renal pelvis and ureters. Exclusion criteria are a known anaphylaxis to iodinated contrast medium, poor renal function (GFR $<45$ $\mathrm{ml} / \mathrm{min}$ ), pregnancy and known history of occluding ureteral disease. Sixty consecutive patients were referred to CTU (40 men, 20 women; age range: 27-82; mean age: 64 years).

Patients were placed randomly into one of the three maneuver groups, group no. 1-3. A total of eight patients were excluded from this study. In group no. $1(\mathrm{n}=18)$ and group no. $2(\mathrm{n}=18)$, two patients each had to be excluded for technical reasons. Additionally, in group no. 2 two patients had to be excluded due to a duplicated ureter $(n=16)$. Because of a status post nephrectomy and a functionless kidney two patients had to be excluded from group no. $3(n=18)$.

\subsection{Imaging}

All patients were examined in supine position with CT scanners (Somatom Sensation 16 and 64; Siemens, Erlangen, Germany). Images were obtained using either $16 \mathrm{~mm} \times$ $1.25 \mathrm{~mm}$ or $64 \mathrm{~mm} \times 0.6 \mathrm{~mm}$ detector collimation and were reconstructed at a slice thickness of $3 \mathrm{~mm}$ with no section overlap. A pitch factor of 1.2 and $120 \mathrm{kVp}$, tube current was patient-dependent based on the body weight with a reference $\mathrm{mA}$ of $200 \mathrm{mAs}$. Prior to CT examination, all patients drank 11 of water, no diuretic medication was given and no voiding was allowed during scan acquisition. All patients received unenhanced images ranging from the upper pole of the kidneys to the bladder (scan 1). Uniformly, $100 \mathrm{ml}$ of intravenous

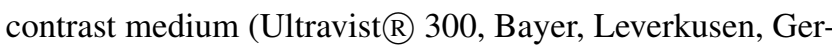
many) was administered with an automatic power injector (Ulrich Medical, Ulm, Germany) using split-bolus technique at defined injection rates: the first bolus of $30 \mathrm{ml}$ of contrast medium was injected at a rate of $2.5 \mathrm{ml} / \mathrm{sec}$; after $180 \mathrm{sec}$, an additional $70 \mathrm{ml}$ of contrast medium bolus was administered followed by $50 \mathrm{ml}$ normal saline injected at $2.5 \mathrm{ml} / \mathrm{sec}$. Uniformly, $90 \mathrm{sec}$ after starting the second injection of contrast agent, a CT scan, extending from the diaphragm to the symphysis pubis, was performed, yielding images in simultaneous nephrographic and excretory phases of enhancement (split-bolus phase) (scan 2). Prior to the third scan, maneuvers described below were performed to improve opacification and distension of the intrarenal collecting system (ICS) and the ureter. The third CT delayed scan was acquired 10 min after injecting the first contrast bolus (see Figure 1) (scan 3).

The patients in the study group 1 had to perform Valsalva maneuvers before and during the third scan. Patients were taught how to perform a Valsalva maneuver, a forceful attempted exhalation against the closed mouth. The patients in study group 2 received anterior lower-abdominal compression using a compression balloon device for about $330 \mathrm{sec}$ after the second acquisition, which was released before scanning. Patients in the third group performed both maneuvers.

Image analysis: All image data were assessed by two independent readers, one with twelve years experience in body radiology and one resident. Both radiologists were blinded with respect to the compression maneuver utilized. They reviewed the axial nephrographic/excretory phase images and late phase images obtained $4.5 \mathrm{~min}$ and $10 \mathrm{~min}$ after IV contrast medium injection, respectively, and multiplanar reformation series using a clinical workstation (GE Medical Systems, Milwaukee, WI). The urinary tract was divided into three segments: ICS, proximal ureter above the iliac crest and distal ureter below the iliac crest. Evaluation of the degree of opacification of the ICS and ureters was performed using a scale ranging from 0 to 2 . A score of 0 indicated no or very minimal opacification; a score of 1 indicated moderate opacification; a score of 2 indicated complete opacification of the ICS or ureter. The distension was measured at the widest point of each given ureteral segment as well as axial diameter of renal pelvis at the ICS. Both, opacification and distension were measured after scan 2 and scan 3 .

\subsection{Statistical methods}

The inter-rater correlation was measured by Pearson's Correlation Coefficient (PCC). Values of PCC $>0.8$ indicated strong agreement and values $<0.5$ indicated poor agreement. Statistical analyses of segmental ureteral distention and segmental ureteral opacification was performed by employing analysis of variance methodologies (ANOVA) with Bonferroni post-hoc corrections, employing segmental ureteral distention and segmental ureteral opacification as the depen- 
dent factor, the type of maneuver, Valsalva, lower abdominal compression and the combination of both as the independent factor, and anatomic location and laterality as additional cofactor. All statistical analyses were performed using SPSS v. 21.0 (PASW Statistics 21; SPSS, Chicago, IL). A $p$-value of $<.05$ was considered to be statistically significant.

\section{Results}

\subsection{Inter-rater correlation}

Regarding the opacification and the distension, a strong interobserver agreement $(0.821)$ was found for the distension and a very strong correlation did exist for opacification (0.944).

\subsection{Starting condition (scan 2)}

To ensure similar starting conditions in all maneuver groups (group 1: Valsalva maneuvers, group 2: lower abdominal compression, group 3: Valsalva + compression) images acquired in the split-bolus phase (scan 2) were taken as starting condition. ANOVA showed no significant difference ( $p$ $=.525$ ) between the three maneuver groups regarding distension. Bonferroni corrections demonstrated no statistical significant differences in distension between groups as a function of segmented location and laterality. Having demonstrated that all three groups showed no significant difference regarding distension and opacification we were able to analyze the effects of each maneuver on each location and on each site of the body.

\subsection{Maneuver assessment}

\subsubsection{Distension}

Mean distension measurements at the level of the renal pelvis showed similar results for each performed maneuver: group 1 (Valsalva group) mean distension was $11.32 \mathrm{~mm}$ (standard deviation $4.3 \mathrm{~mm}$ ), group 2 (compression group) $13.3 \mathrm{~mm}$ (standard deviation $6.2 \mathrm{~mm}$ ) and group 3 (combination of Valsalva and compression) $13.24 \mathrm{~mm}$ (standard deviation $9.4 \mathrm{~mm}$ ), respectively. Detailed measurements are presented in Table 2. However, those measurable differences in size showed no significant difference in chosen maneuvers $(p=$ .187), if patient's site and anatomic location were taken as cofactors. However, comparing the distension during Valsalva and the combination of Valsalva and compression a significant increase in distension was found $(<.05)$; location was found as a significant covariate.

\subsubsection{Opacification}

Opacification was rated at the three given locations. At the level of the renal pelvis group 1 (Valsalva group) mean opacification was 1.93 (standard deviation 0.3), group 2 (compression group) 1.83 (standard deviation 0.5 ) and group 3 (combination of Valsalva and compression) 1.81 (standard deviation 0.5), respectively. Detailed grading results are presented in Table 3. No significant change in opacification scoring was found for the chosen maneuver $(p>.5)$. Location as a covariate is a significant parameter $(p<.5)$.

As shown in Table 1, Bonferroni correction showing no significance for each maneuver group.

Table 1. Bonferoni correction

\begin{tabular}{|c|c|c|c|c|c|c|}
\hline \multirow[b]{2}{*}{ Maneuver } & & \multirow{2}{*}{$\begin{array}{l}\text { Mean } \\
\text { Difference }\end{array}$} & \multirow{2}{*}{$\begin{array}{l}\text { Std. } \\
\text { Error }\end{array}$} & \multirow[b]{2}{*}{ Significance } & \multicolumn{2}{|c|}{ 95\% Confidence Interval } \\
\hline & & & & & Lower & Upper \\
\hline \multirow{2}{*}{ Control Valsalva } & Control Compression & -175 & 574 & 1000 & -155 & 120 \\
\hline & Control Valsalva \& Compression & -.614 & .557 & .811 & -1.95 & .72 \\
\hline Control & Control Valsalva & .175 & .574 & 1.000 & -1.20 & 1.55 \\
\hline Compression & Control Valsalva \& Compression & -.439 & .572 & 1.000 & -1.81 & .93 \\
\hline Control Valsalva & Control Valsava & .614 & .557 & .811 & -.72 & 1.95 \\
\hline \& Compression & Control Compression & .439 & .572 & 1.000 & -.93 & 1.81 \\
\hline
\end{tabular}

Table 2 shows mean measurements $(\mathrm{mm})$ of distension at the three positions in all three maneuver groups. Group 1= Valsalva, group $2=$ Compression, group $3=$ Valsalva and Compression. $\mathrm{N}=$ number.

Table 3 shows mean grading of opacification at the three positions in all three maneuver groups. Group $1=$ Valsalva, group 2 = Compression, group $3=$ Valsalva and Compression. $\mathrm{N}=$ number. Scale ranging from 0 to 2 . $0=$ no or very minimal opacification; 1 = moderate opacification; 2 =

Published by Sciedu Press complete opacification of the ICS or ureter.

As shown in Figure 1, an unenhanced scan followed by contrast medium administration, split into two bolus injections. After finishing the second bolus injection, the second scan (split-bolus CT) was acquired with a total time delay of 270 sec after first injection. After this scan the three different types of maneuvers were carried out and a third scan (late phase CT) was performed after a total of $600 \mathrm{sec}$. 
Table 2. Mean measurements of distension

\begin{tabular}{lllll}
\hline Group & & Mean & Std. deviation & N \\
\hline \multirow{3}{*}{1} & Renal Pelvis & 11.32 & 4.32 & 72 \\
& Proximal Ureter & 5.26 & 2.50 & 72 \\
& Distal Ureter & 4.89 & 3.96 & 72 \\
\\
& Renal Pelvis & 13.33 & 6.15 & 64 \\
& Proximal Ureter & 5.27 & 2.18 & 64 \\
& Distal Ureter & 5.05 & 2.16 & 64 \\
& Renal Pelvis & 13.24 & 9.42 & 72 \\
& Proximal Ureter & 6.53 & 2.83 & 72 \\
& Distal Ureter & 4.68 & 2.54 & 72 \\
\hline
\end{tabular}

Table 3. Mean grading of opacification

\begin{tabular}{lllll}
\hline Group & & Mean & Std. Deviation & N \\
\hline \multirow{3}{*}{1} & Renal Pelvis & 1.93 & 0.31 & 72 \\
& Proximal Ureter & 1.43 & 0.75 & 72 \\
\multirow{3}{*}{2} & Distal Ureter & 1.18 & 0.88 & 72 \\
& Renal Pelvis & 1.83 & 0.49 & 64 \\
& Proximal Ureter & 1.48 & 0.73 & 64 \\
3 & Distal Ureter & 1.36 & 0.82 & 64 \\
& Renal Pelvis & 1.81 & 0.52 & 72 \\
& Proximal Ureter & 1.53 & 0.69 & 72 \\
\hline
\end{tabular}

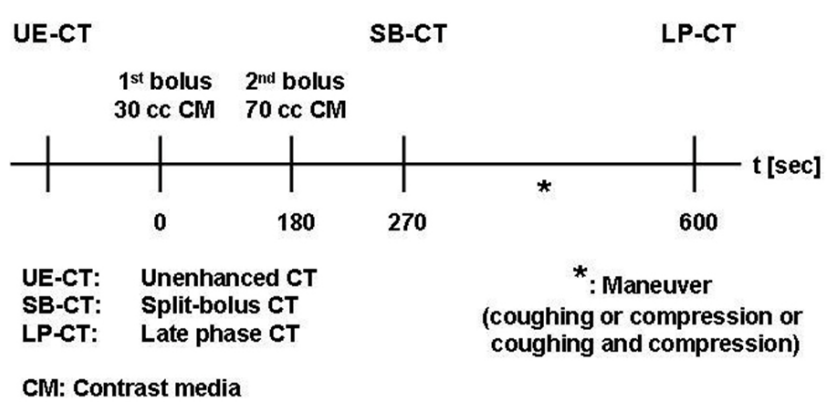

Figure 1. Schematic view of scanning protocol

\section{Discussion}

Currently, no existing CTU protocol is universally applied though recent effort has been aimed to optimize excretory phase urinary tract opacification and distension to better outline the lumen of the ureter with excreted contrast. Representative CTU protocols contain either a three-phase acquisition with a single-bolus injection or a two-phase acquisition with a two bolus-injection (split-bolus). ${ }^{[6-10]}$

Our scan protocol consists of an unenhanced scan (scan 1), followed by a split-bolus phase (scan 2), as advocated by different study groups. ${ }^{[4,11-13]}$ Scan 2 was followed by supportive maneuvers and a late phase CT (scan 3). $10 \mathrm{~min}$ after the first IV injection of contrast medium, the third scan was obtained, as a longer time delay has been described as preferable regarding distension and opacification of the distal urinary tract. ${ }^{[6,14]}$

Patients were placed randomly into one of the three maneuver groups. After the split-bolus CT (scan 2) we could demonstrate that no significant difference exists among the groups regarding distention and opacification. Though the longer scan time and associated interscan delays by themselves improve distension and opacification of the urinary tract, we increased abdominal pressure after the split-bolus phase to further improve distension and opacification of the urinary tract. Valsalva maneuvers and temporary external abdominal compression are already established and used both in IVU und CTU. ${ }^{[3-5]}$ Elevated abdominal pressure should improve opacification and distension of the ICS and proximal ureter while being applied and of the mid and distal ureter upon release.

Valsalva maneuvers are an easy technique which is almost always feasible in patients with unexplained hematuria.

Another maneuver to increase intraabdominal pressure was realized by temporarily applying an external lower abdom- 
inal compression. This method is already well established in IVU and has been evaluated by many investigators in CT. ${ }^{[3-6,8]}$ External compression is not as straight forward implementable compared to Valsalva maneuvers and it is even impractical in patients suffering from abdominal pain, recent abdominal surgery or known abdominal aortic aneurysm.

In scan 3 we observed some measurable differences in distension size without showing any significance in chosen maneuvers, if patients' site and anatomic location were taken as cofactors. The combination of Valsalva and compression showed significant higher distension sizes if location was taken as covariate. Our numbers suggest to perform compression if only one maneuver should be applied. Similar results were shown by Caoili et al. who compared abdominal compression during CT, scanned 150 and $300 \mathrm{sec}$ after IV injection, and patients without compression, to assess the effect of opacification and distension at the proximal renal collecting system. They also demonstrated that the maximal diameter increases significantly at later scan time by using compression. Additionally, a significantly larger distension and a more homogeneous opacification were seen for compression compared to the group without compression at $300 \mathrm{sec}$ after IV injection. ${ }^{[15]}$ In another study Caoili et al. confirmed that compression could improve distension and opacification of the ICS and the proximal ureter, however none of the differences were statistically significant. ${ }^{[6]}$

Our results showed no significant change in opacification for chosen maneuvers if location was chosen as a cofactor. Anatomically given the location is a significant parameter if taken as a covariate, the distal ureter is the most challenging segment to opacify.

Poor opacification was also described by others with an absence of opacification in $2 \%$ and $28 \%$, respectively in the distal segments. ${ }^{[7,18]}$ McNicholas et al. showed that the distal ureter was significantly less opacified on supine CTU than IVU. However, opacification of the distal ureter was best visualized with compression CT which was as good as with IVU. ${ }^{[3]}$

A further method improving depiction of urinary tract is hydration prior to imaging. In our study, CT examinations of all patients were preceded by ingestion of 11 of water. Oral hydration with water is without any additional cost and promotes diuresis and thus distension of the urinary tract; however caution is required for patients with limited liquid intake. Several investigators used similar regimes describing that 20 min prior to scanning patients ingested up to 11 of tap water ${ }^{[16-18]}$ or "six cups" of water during $60 \mathrm{~min}$ before scanning. ${ }^{[19]}$ Saline infusion might be used as an alterna- tive. ${ }^{[6,20,21]}$ However, some studies showed no particular benefit for saline injection or administration of oral water. ${ }^{[22]}$ Oral hydration decreases the attenuation values in the urinary tract at the excretory phase, but improves the continuous opacification of the ureter. ${ }^{[23]}$

\section{Limitations}

There were limitations to our study. Patients were placed consecutively into the groups. Therefore unforeseen unidentified variations might exist within the groups, although clinical indication were the same for all patients and after split-bolus CT no significant difference among the groups was found regarding distention and opacification. We decided not to compare different scan protocols within the same patient to lower the radiation dose to a minimum. No control group was established as different papers have already stated an insufficient visualization of the distal ureter in up to $33 \%{ }^{[6]}$

Only small groups of patients were studied, larger trials are desirable. The three-grade opacification scoring system is limited in terms of sensitivity to nuances. As supportive maneuvers we used Valsalva maneuvers and temporary abdominal compression by inflation of a balloon placed over the lower abdomen. Intraabdominal pressure depends on body mass, positioning of the balloon and even patients' compliance to generate Valsalva conditions. It would be difficult to implement objective measurements of any supportive maneuver.

\section{Conclusion}

A CTU protocol including a late phase scan with prior application of compression, Valsalva maneuvers or a combination of both showed beneficial effects for the distension and opacification, a significant difference was found by taking location as a covariate.

\section{ETHICAL APPROVAL}

All procedures performed in studies involving human participants were in accordance with the ethical standard of the institutional and/or national research committee and with the 1964 Helsinki declaration and its later amendments or comparable ethical standards.

\section{INFORMED CONSENT}

Informed consent was obtained from all individual participants included in the study.

\section{Conflicts of InTERest Disclosure}

The authors have no conflict of interest related to this publication. 


\section{REFERENCES}

[1] Van Der Molen AJ, Cowan NC, Mueller-Lisse UG, et al. CT urography: definition, indications and techniques. A guideline for clinical practice. European Radiology. 2008; 18(1): 4-17. PMid:17973110 https ://doi.org/10.1007/s00330-007-0792-x

[2] Ziegler J. Bedeutung und Technik der Ureterkompression bei der Ausscheidungspyelographie. Dtsch med Wochenschr. 1930; 56(42): 1772-1775. https://doi .org/10.1055/s-0028-1125999

[3] McNicholas MM, Raptopoulos VD, Schwartz RK, et al. Excretory phase CT urography for opacification of the urinary collecting system. AJR. American Journal of Roentgenology. 1998; 170(5): 1261-1267. PMid:9574598 https ://doi.org/10.2214/ajr.17 0.5 .9574598

[4] Chow LC, Sommer FG. Multidetector CT urography with abdominal compression and three-dimensional reconstruction. AJR. American Journal of Roentgenology. 2001; 177(4): 849-855. PMid:11566687 https://doi.org/10.2214/ajr.177.4.1770849

[5] Heneghan JP, Kim DH, Leder RA, et al. Compression CT urography: a comparison with IVU in the opacification of the collecting system and ureters. Journal of Computer Assisted Tomography. 2001; 25(3): 343-347. PMid:11351181 https://doi.org/10.1097/00 004728-200105000-00003

[6] Caoili EM, Inampudi P, Cohan RH, et al. Optimization of multidetector row CT urography: effect of compression, saline administration, and prolongation of acquisition delay. Radiology. 2005; 235(1): 116-123. PMid:15716388 https://doi.org/10.1148/ radiol. 2351031085

[7] Kekelidze M, Dwarkasing RS, Dijkshoorn ML, et al. Kidney and urinary tract imaging: triple-bolus multidetector CT urography as a one-stop shop-protocol design, opacification, and image quality analysis. Radiology. 2010; 255(2): 508-516. PMid:20160002 https://doi.org/10.1148/radiol.09082074

[8] Caoili EM, Cohan RH, Korobkin M, et al. Urinary tract abnormalities: initial experience with multi-detector row CT urography. Radiology. 2002; 222(2): 353-360. PMid:11818599 https : //doi.org/10.1148/radiol.2222010667

[9] Akbar SA, Mortele KJ, Baeyens K, et al. Multidetector CT urography: techniques, clinical applications, and pitfalls. Seminars in ultrasound, CT, and MR. 2004; 25(1): 41-54. https://doi .org/10.1053/j . sult. 2003.11.002

[10] Wolin EA, Hartman DS, Olson JR. Nephrographic and pyelographic analysis of CT urography: differential diagnosis. AJR. American Journal of Roentgenology. 2013; 200(6): 1197-1203. PMid:23701053 https://doi.org/10.2214/AJR.12.9692

[11] Sanyal R, Deshmukh A, Singh Sheorain V, et al. CT urography: a comparison of strategies for upper urinary tract opacification. European Radiology. 2007; 17(5): 1262-1266. PMid:17039364 https://doi.org/10.1007/s00330-006-0462-4

[12] Chai RY, Jhaveri K, Saini S, et al. Comprehensive evaluation of patients with haematuria on multi-slice computed tomography scanner: protocol design and preliminary observations. Australasian Radiology. 2001; 45(4): 536-538. PMid:11903196 https ://doi .org/10 $.1046 / j .1440-1673.2001 .00978 . x$
[13] Maher MM, Kalra MK, Rizzo S, et al. Multidetector CT urography in imaging of the urinary tract in patients with hematuria. Korean Journal of Radiology: Official Journal of the Korean Radiological Society. 2004; 5(1): 1-10. PMid:15064553 https ://doi.org/10 .3348/kjr.2004.5.1.1

[14] Curic J, Vukelic-Markovic M, Marusic P, et al. Influence of bladder distension on opacification of urinary collecting system during CT urography. European Radiology. 2008; 18(5): 1065-1070. PMid:18274759 https : //doi.org/10.1007/s00330-008-085 8-4

[15] Caoili EM, Cohan RH, Korobkin M, et al. Effectiveness of abdominal compression during helical renal CT. Academic Radiology. 2001; 8(11): 1100-1106. https ://doi.org/10.1016/S1076-6332(03 ) $80721-3$

[16] Cowan NC, Turney BW, Taylor NJ, et al. Multidetector computed tomography urography for diagnosing upper urinary tract urothelial tumour. BJU International. 2007; 99(6): 1363-1370. PMid:17428251 https://doi.org/10.1111/j.1464-410X.2007.06766.x

[17] Silverman SG, Akbar SA, Mortele KJ, et al. Multi-detector row CT urography of normal urinary collecting system: furosemide versus saline as adjunct to contrast medium. Radiology. 2006; 240(3): 749755. PMid:16926326 https://doi.org/10.1148/radiol. 240 3050233

[18] Kawamoto S, Horton KM, Fishman EK. Opacification of the collecting system and ureters on excretory-phase CT using oral water as contrast medium. AJR. American Journal of Roentgenology. 2006; 186(1): 136-140. PMid:16357392 https://doi.org/10.2214/ AJR. 04.1457

[19] Raptopoulos V, McNamara A. Improved pelvicalyceal visualization with multidetector computed tomography urography; comparison with helical computed tomography. European Radiology. 2005; 15(9): 1834-1840. PMid:15761715 https ://doi.org/10.1007/ s00330-005-2699-8

[20] McTavish JD, Jinzaki M, Zou KH, et al. Multi-detector row CT urography: comparison of strategies for depicting the normal urinary collecting system. Radiology. 2002; 225(3): 783-790. PMid:12461262 https://doi.org/10.1148/radiol.2253011515

[21] Sun H, Xue HD, Liu W, et al. Effects of Saline Administration, Abdominal Compression, and Prolongation of Acquisition Delay on Image Quality Improvement of CT Urography. Chinese Medical Sciences Journal $=$ Chung-kuo i hsueh k'o hsueh tsa chih/Chinese Academy of Medical Sciences. 2013; 27(4): 201-206.

[22] Sudakoff GS, Dunn DP, Hellman RS, et al. Opacification of the genitourinary collecting system during MDCT urography with enhanced CT digital radiography: nonsaline versus saline bolus. AJR. American Journal of Roentgenology. 2006; 186(1): 122-129. PMid:16357390 https : //doi.org/10.2214/AJR.04.1835

[23] Szolar DH, Tillich M, Preidler KW. Multi-detector CT urography: effect of oral hydration and contrast medium volume on renal parenchymal enhancement and urinary tract opacification-a quantitative and qualitative analysis. European Radiology. 2010; 20(9): 2146-2152. PMid:20383638 https : //doi .org/10.1007/s00330-010-178 $5-8$ 